\title{
Putting beach slope prediction into perspective
}

\author{
R.J. Jewell Australian Centre for Geomechanics, The University of Western Australia, Australia
}

\section{Abstract}

The storage capacity of any given tailings storage facility (TSF) is a function of the volume available for the tailings for which the geometry of the final upper surface of the tailings is most important. One of the advantages that can be obtained from thickening tailings prior to discharge is that the tailings can be stacked at a steeper beach angle than is obtainable with conventional low density slurries. However, despite ongoing efforts by a number of parties, there is at present no universally accepted method available for the accurate prediction of tailings beach slopes.

From an operating perspective, the tailings parameters relevant to the resulting beach slope can fluctuate significantly in the product delivered to the TSF and it is relatively well understood that the final beach slope can be manipulated by changes to disposal techniques. There are also newly emerging technologies that will enhance the dewatering of tailings at discharge and hence expedite the consolidation of the tailings that could well facilitate the development of steeper beach slopes. Hence, while the beach slope is an important parameter for the design of a TSF suitable to contain any given mass/volume of tailings, it may well be more important to understand the means by which the tailings parameters may be modified or the beach slope manipulated by the operators to achieve a reasonable design value than to develop a generic beach slope prediction model.

\section{$1 \quad$ Introduction}

The concept of thickening tailings before discharge was first promoted by Eli Robinsky with the objective of reducing the height and hence the loading of the confining embankments of a TSF on underlying sensitive Leda clays in Ontario. In order to achieve that, the thickened tailings needed to reach equilibrium at a steeper slope after discharge than achieved with conventional low density tailings slurry so that a greater mass of tailings could be stored within the area encompassed by the confining embankment. Since then, the "holy grail" for designers has been to determine how to predict the beach slope for the tailings to be discharged at any given operation.

Considerable effort has been expended by many service providers and researchers into trying to derive means by which beach slope prediction could be encoded. However, to this date the search for a generally recognised method of accurately predicting tailings beach slopes has yet to be fulfilled. Ultimately all current designs must utilise beach slope predictions reflecting the expert opinion of the designer based upon experience and an understanding of the slopes generated on projects involving similar ore bodies, throughput, tailings properties, topography and environment.

The basis for three very different methods representing the current status of different ongoing efforts over the past decade and longer by four groups of researchers and practitioners who have, to some extent, at least published on this topic in recent years, have been outlined in this paper. However, there are as yet no independently verified projects or published references to projects on which a Class A prediction has been validated for any of these methods. Until that occurs, and any claims by the proponents to the contrary, the current predictions should only be viewed as 'best practice' predictions to be used as one of a range of indicators to be used by experienced practitioners in order to suggest a range of slope angles likely to result for any given operation.

What is clear however, is that the variations inherent in any mining operation over the long term, including the properties of the orebody, plant throughput, thickener underflow density, etc., can result in 
considerable fluctuation in the properties of the tailings discharged into a TSF. The result is that specific parameters used in any design may change over time and nullify any beach slope prediction. Accordingly, it may well be that greater attention should be paid to means by which the properties of the tailings delivered to and the discharge techniques into the TSF may be modified to achieve any desired design slope, than to the accuracy of the original prediction.

This paper examines the current situation with the objective of putting the quest for a method for the accurate prediction of beach slopes into perspective.

\section{Background to TSF development}

In early mining operations the tailings generated were allowed to spread unchecked into the nearest available gully, which may or may not have been a waterway of some description. Subsequently, a number of different means of disposing of the waste product have been developed and practiced for reasons including:

- to constrain the extent of the area covered by tailings

- to enhance the recovery of process water

- to retain the tailings to permit reprocessing at some later stage

- to limit the potential for the release of contaminants and damage to the environment.

Early mining operations generally had a low throughput and even when deposited at low density there was mostly ample time for the tailings slurry to dry out and stabilise as the tailings built up in the TSF. As the scale of mining operations has increased and the desire to fit more tailings onto any given footprint and reduce transport costs, the rate of buildup of tailings into TSFs has increased. To facilitate the recovery of mineral from the ore, commination processes are producing finer tailings which retard the drying process and all of these factors are resulting in the TSF embankments now often retaining extensive deposits of saturated tailings. There have unfortunately been major failures of above ground TSFs due to surface water overtopping the embankment and the subsequent liquefaction of the tailings, which has resulted in many deaths and considerable environmental damage. The desire to provide safer storage facilities is just one of the factors leading towards the adoption of thickening tailings technology.

The beach slopes of conventional tailings discharged as a low density slurry have traditionally been very shallow, varying in slope from around $1 \%$ at the point of discharge where the coarse fraction was deposited down to near zero at the toe of the slope where the very finest fractions come to rest at the decant pond as a result of segregation. There is a requirement in these facilities to provide a freeboard on the confining embankments sufficient to retain the decant pond containing both the supernatant water and rainfall runoff as well as any displacement of tailings due for example to liquefaction. This requires that the confining embankments are constructed to a level above the top of the beach and in most cases steepening up the beach slope would actually be detrimental to the available storage volume of the TSF.

With the introduction of thickened tailings technology, the tailings are transported and discharged into the TSF at a higher consistency. As a result, the tailings release less supernatant water on discharge and have higher yield shear stresses that often result in steeper beach slopes. The tailings are still susceptible to liquefaction but under normal circumstances a storage facility can be designed to safely store tailings above the embankment height and therefore being able to predict the slope of the beach has significance in terms of estimating the available storage volume.

It is four decades since Eli Robinsky first espoused the concept of thickening tailings to reduce the height (and base loading) of confining embankments for a central discharge scheme at the Kidd Creek mine in Ontario (Robinsky, 1999). Since that first unsuccessful attempt, the capability of the thickeners and pumping equipment has developed to the extent that quite high yield stress slurries can now be produced and transported to discharge. The stack geometry controls the storage for a given footprint and this has led 
to many innovative and individual designs for TSFs taking advantage of the natural topography of the available sites, as is evident in the many published references to projects around the world.

\section{$3 \quad$ Beach slope prediction experience}

Robinsky based his beach slope predictions on the slopes obtained in flume tests using representative tailings samples and it appears from the literature that many other practitioners since then have based their predictions on similar data. There are also a number of references in the available literature to operations in which no basis is provided for the beach slopes predicted; some quasi rational predictive method has been outlined or, the prediction has been based upon nothing but experience with other operations having similar geometry, tailings parameters, throughput, etc. Unfortunately, experience has shown that the beach slopes developed in the field have never even nearly approached the slopes developed in laboratory scale flume testing and indeed, anecdotal evidence suggests that there are many examples of operations in which the design predictions for tailings beaches have not been realised regardless of the method of prediction.

One of the challenges faced in expanding on this topic is that in many cases the original predictions are not readily available. However, in a recent paper (Jewell, 2010) this issue was discussed in terms of a design prediction for a major, but unnamed copper project as follows:

The plant was to consist of two banks of very large diameter deep cone thickeners, each thickener with a design throughput of the order of 12,000-15,000 t/day. The thickeners were to be established at an elevation such that the tailings were expected to be discharged primarily under gravity with limited shear thinning and to spread out across the TSF footprint at beach slopes of somewhere between 5 and $8 \%$.

There was no way in which these predictions could be challenged, except to note that beach slopes of that magnitude had not been developed on a continuous basis and/or at that rate of throughput at any operation involving deep cone thickeners anywhere in the world at that time (or since). On the basis of experience with similar thickened tailings operations it appeared to be more prudent to consider a range of beach slopes of between say 2 and $5 \%$ for the design process.

In justifying that lower beach slope, it appeared prudent to consider the Miduk copper project in Iran for which the design prediction for supergene as listed by Williams et al. (2006) was $3.5 \%$ and it was expected that the underflow percent solids and beach angle would increase slightly as the change to hypogene ore occurred. The 2006 paper indicates that the beach slopes measured during a survey in August 2005 were around 2.3 to $2.4 \%$ and the beach slope listed in the 2008 paper for the project was $2.5 \%$. Both projects involved copper tailings thickened by deep cone (very high density) thickeners with a high daily throughput and were considered to be sufficiently similar to justify an expectation that the tailings would form similar beach slope angles.

In a relatively recent paper (Williams et al., 2008) the authors have compiled a list providing relevant details of 34 thickened tailings operations worldwide and this remains the most comprehensive source of information of these operations yet published. These observations have confirmed the view that the expectation of developing beach slopes of 5 to $10 \%$ with raw thickened tailings slurry is unrealistic. Only one mine site is shown on the list as achieving a beach slope in excess of $5 \%$ and that is the Bulyanhulu operation in Tanzania which is listed as having a maximum beach slope of $10 \%$. Bulyanhulu is however almost unique in that it is an underground mine and all of the tailings are first filtered to a filter cake for incorporation into the mine backfill operations. Any excess is first conditioned back to a high (and very uniform) consistency and pumped to an above ground TSF and discharged at a low rate through multiple towers to achieve the steep beach angles. Even on that project there is anecdotal evidence to suggest that scouring and wash outs have occurred on the beaches when the tailings were conditioned back to a lower consistency than planned before discharge.

To be completely fair and rational about any discussion about beach slopes, one needs to be aware that in many cases the poor correlation between prediction and practice can also relate to the discrepancy 
between fundamental design assumptions used as inputs to beach slope models, and the conditions that actually apply during operations. In a recent paper (Seddon and Fitton, 2011), the authors note that even in relatively stable operations there will be unavoidable variations in flow rate and rheology which must be taken into consideration in the design. For example the type or mix of ore may also vary during the mine life with impacts on the resulting slurry rheology, as well as conscious decisions regarding the operation of thickeners that result in a change in solids concentration and slurry rheology. Finally of course, site operations and depositional methods can have a significant influence on local flow rates in the tailings, and hence beach slope.

\section{$4 \quad$ Beach slope prediction methods}

A comprehensive review and comparison of the methods of beach slope prediction and control of stack evolution was presented in a paper by Simms et al. (2011), to which should be added the work presented independently by Li (2011) at the same forum. These papers presented the current status of different ongoing efforts over the past decade and longer by four groups of researchers and practitioners who have to some extent at least published on this topic in recent years. In effect there are to our knowledge three very different methods being pursued.

Simms et al. (2011) wrote that a model largely developed by Tim Fitton (building on a decade of work by ATC Williams), integrates non-Newtonian open channel flow and sediment transport in which it is assumed that the maximum beach slope corresponds to the slope of a channelised tailings flow in which the tendency for erosion and sedimentation reaches equilibrium. On the other hand, Simms wrote that the basic premise of the method developed by Gordon McPhail is that the beach slope profile is related to the dissipation of the energy of the tailings (now quantified as 'stream power') as they move downslope in a flow channel. McPhail first formulated this by applying an 'entropy' based approach to the problem of assessing the attainment of equilibrium as the tailings are discharged and flow down the beach. Allan Li (2011) wrote that in his approach, "the model is developed based on solid mechanics and fluid mechanics principles satisfying limit equilibrium conditions and energy conservation".

In order to apply each of the models and to predict beach slopes, the proponents need input parameters for the operation (e.g. throughput and thickener underflow design density) and properties of the tailings (e.g. specific gravity, particle size distribution and rheological test data including yield stress and viscosity). In addition, Li (pers. comm., January 2012) looks for topographical data for the proposed TSF and the number of proposed spigots in a multiple discharge system, while McPhail (pers. comm., January 2012) suggests that beach trial testing at a moderate scale be undertaken to obtain additional data used in his formulation.

\section{1 Validation of beach slope prediction models}

To our knowledge, there are as yet no independently verified projects or published references to projects on which a Class A prediction has been validated for any of these approaches. Until that occurs, and any claims by the proponents to the contrary the current predictions should only be viewed as "best practice" predictions to be used as one of a range of indicators to be used by experienced practitioners in order to suggest a range of slope angles likely to result for any given operation.

The three different approaches to beach slope prediction introduced above were subjected to vigorous discussion at a workshop expressly convened for the purpose and held in conjunction with the Paste 2011 seminar and attended by 65 practitioners. There were a number of outcomes of the workshop that are relevant to this discussion. For example:

- Laboratory scale flume testing is not a viable method for predicting a beach slope. Clearly, the slopes generated in both flume tests and in the field are functions of the yield shear stress of the tailings, but not all of the factors influencing the beach slope in the field are "in play" in the flume (and vice versa). Slopes developed in the flume are demonstrably steeper than in the field and to date no valid relationship between the two has been established. 
- Field observations suggest that beach slopes achieved to date in the field for thickened tailings derived as thickener underflow have rarely exceeded say 2.5 to $3 \%$ over an extended period. Hence, design predictions steeper than that for conventional thickened tailings operations should be treated with caution and indeed for most operations beach slopes of 1.5 to $2 \%$ will be more likely.

- There is a need for further work to validate whether any one (or all) of these three different design approaches will eventually be capable of accurately predicting beach slopes for tailings. To be generally accepted, this will ultimately need an approach in which Class A predictions are made on the basis of input parameters (ideally standard test data) and then compared with actual slopes generated in the field once operations are underway.

- There is a need for data from field operations suitable for the undertaking of Class A predictions. The industry is to be urged to facilitate data being made available to the different research teams to enable the undertaking of comparative studies.

- The final beach slope can be manipulated by modifying the thickener underflow and by changes to disposal techniques and attention to these operating issues is required for any given beach slope to be achieved.

\section{2 Modifying tailings properties and operations to increase beach slopes}

Seddon and Fitton (2011) have attempted to quantify the effect that relatively minor variations in the mineralogy of the orebody, the geotechnical or rheological parameters of the tailings or of changes to the operation, such as rate of throughput or discharge technique, can have on the resulting beach slope. One objective of the authors appears to have been to provide an explanation as to why the upper surface of thickened tailings beach slopes are almost inevitably steeper near the discharge point than at the toe of the slope. Thickened tailings would not be expected to segregate and hence should produce linear beaches rather than having a concave upper surface as is commonly observed. What is evident from the paper however is that it would appear prudent to treat any beach slope prediction as an indicative value that may be manipulated by subsequent operating practices.

In practice it is clear that the rate of discharge of tailings at discharge can have an effect on the resulting beach slope. The greater the volume and velocity of discharge, the greater the tendency for the tailings to scour flow channels, to flow further down the slope and to produce a flatter beach. Reducing the volume and velocity of discharge at any given location by means of dividing the flow between a number of discharge points (e.g. spigots) has been shown to produce laminar flow resulting in less scouring and steeper beach slopes and introducing multiple spigots is the most common discharge technique in general use to achieve this result.

In addition to modifying the rate of discharge of the tailings, arguably the most efficient technique that might be adopted in order to increase the beach slopes at discharge is to increase the yield shear stress of the discharged tailings. This may be achieved by one or a combination of:

- Modifying the thickener operations to achieve the design density and hence shear stress in an underperforming thickener underflow when appropriate.

- Increasing the efficiency of the thickener to produce a higher underflow density than originally designed, if that is possible.

- Altering the components of the system of transport of the tailings from the thickener to the TSF to reduce the losses to shear stress in the pumps and pipeline.

- Efficient management of an appropriate deposition strategy.

In addition to the techniques listed above there is another concept still in the early stages of development that may well prove to be very positive in terms of reducing the variability in the rheology of the tailings at 
discharge and increasing the probability of being able to produce a consistently higher beach slope. This concept is already proving to be of particular value in treating the fine grained fraction (slimes) of tailings (and also potentially to the total tailings stream) and involves the injection of a polymer into the tailings at discharge as is explained in more detail below.

\section{Polymer modification of tailings at discharge}

The impetus to find an improved means of treating the fine grained fraction (slimes) of tailings has recently resulted in the promotion of polymers that when injected into the tailings at discharge create a flocculated structure that has the effect of enhancing the drainage of water from the fines (as supernatant water) and consequently expediting the consolidation of the slimes. This technique is currently being used on trials with very low density slimes (mature fine tailings) at oil sands operations in Alberta as well as in trials at bauxite operations in Western Australia.

The alumina industry in Australia has traditionally separated the coarse and fine fractions of the tailings derived from the bauxite, with the sand fraction being used to construct embankments for above ground TSFs and other construction uses, while the slimes have been thickened to a paste and discharged into the TSFs. The slimes are discharged in relatively thin lifts and allowed to dry through evaporation before subsequent lifts are placed. The drying time varies with the time of the year, but an average time to dry a $300 \mathrm{~mm}$ thick lift appears to be around 100 days. Current trials with the polymer injection concept are showing that the enhanced release of supernatant water is enabling the movement of equipment and personnel onto the "red mud' tailings within some 20 or so days, which will significantly reduce the cost of tailings storage through a reduced time between lifts and reduced TSF area requirements.

At least one magnetite iron ore mine in Western Australia is understood to be in the process of applying the technique on a very large scale and another is still in the design phase. The objective for these operations is to increase the beach slope that can be achieved for the tailings in the TSF (as a consequence of the increased yield stresses that will result from the enhanced drainage and expedited consolidation of the discharged tailings). These magnetite tailings contain very little clay sized particles and essentially no clay minerals and will not be classified into coarse and fine fractions for processing. Accordingly, the (rock flour) tailings will contain the total range of particle sizes and a successful application will demonstrate that the technique can treat more than the slimes fraction alone.

A limited search for references has unearthed papers relating to the injection of a polymer into tailings at discharge that refer to two main polymer manufacturing companies - BASF (CIBA) and SNF. Four papers, Kaiser et al. (2006), Adkins (2008), Daubermann and Foldvari (2009) and Berger et al. (2011) relate to the use of the CIBA/BASF product RHEOMAX; one paper on modifying coal washings with an SNF polymer by Bembrick (2008) and a paper by Wells et al. (2011) on the modification of MFT fines at Suncor with an SNF polymer. Another paper on MFT oil sands tailings at the Muskeg River Mine in Northern Alberta by Matthews et al. (2011) provides interesting and very similar data, but without any details of the polymer involved.

The paper by Wells indicated that in the Suncor trials the effectiveness of the polymer in increasing yield stress and enhancing the drainage of water from the tailings is closely related to the extent of shearing to which the injected tailings are subjected and that this is an aspect that is still under investigation. Very importantly, in this trial and the one conducted at Muskeg River, the supernatant water was reported to be essentially clear and thus suggested that the water had been effectively separated from the fine particles in the slimes.

At least two trials have been conducted in Western Australia on fine grained alumina tailings involving inline injection of polymers into tailings at discharge. The 'red mud' tailings produced at such alumina operations generally dry out and consolidate slowly, being both very fine grained and having a significant clay content. The relatively rapid drying, increased yield stress and lack of segregation down slope being observed in the local trial is encouraging as these alumina fine grained tailings are primarily clay minerals. 
These references suggest that injecting a polymer prior to discharge to modify the structure of the tailings is a technique that was probably first undertaken within the last five or so years, which might explain why so few references to such applications have been identified. Most of the papers found have been authored by personnel employed by the chemical companies, or relate to trials set up in conjunction with the chemical companies to examine the viability of the technique and suggest that there are as yet few if any operations already applying the technique in the field (or willing and authorised to publish their findings).

Further investigation would be required in order to ensure that this concept would be suitable for any given operation. The technique appears to have first evolved as a means of enhancing the release of supernatant water from tailings at discharge (and in the process clarifying the water) which could be very valuable for example, for dewatering the slimes in red mud, oil sands, mineral sands and similar operations and possibly facilitating the return of slimes directly to backfill. However, if the increase in yield stress at discharge, due to expedited consolidation of the tailings also facilitates an increase in beach slope, this concept could well revolutionise tailings management around the world where the increase in costs for the polymer and upgraded management that will necessarily be associated with the technique can be justified.

\section{Conclusions}

A comprehensive review and comparison has been reported of three known but very different methods for the estimation of a beach slope in tailings deposits. These are essentially techniques developed by different consultants and used by them in-house on their design projects, but as yet no generally accepted method of accurately predicting tailings beach slopes is available.

There is a need for further work to validate whether any of these three or other design approaches will eventually be capable of accurately predicting beach slopes for tailings. Until that occurs, the current predictions should only be viewed as 'best practice' predictions to be used as one of a range of indicators to be used by experienced practitioners in order to suggest a range of slope angles likely to result for any given operation.

Laboratory scale flume testing is not a viable method for predicting a beach slope. Slopes developed in the flume are demonstrably steeper than in the field and to date no valid relationship has been established between the two.

There appears to be a relatively poor correlation to date between beach slopes achieved in practice and the initial design values. This may be the direct result of imprecise slope prediction methods, but there are likely to be at least as many cases where the poor correlation is due to the discrepancy between fundamental design assumptions used as inputs to beach slope models, and the conditions that actually apply during operations.

While it will be important to pursue the concept of developing a validated, uniformly accepted and accurate method for beach slope prediction, it is also important to accept that any prediction will only be as relevant as the design parameters which will inevitably vary with time on all operations. However, the beach slope can be modified during operations by a number of techniques and it may be more important to recognise and adopt those techniques as appropriate to massage the beach slope in the field to meet the design profile than to make an accurate prediction in the first place.

In practice it is clear that the rate of discharge of tailings at discharge can have an effect on the resulting beach slope. Reducing the volume and velocity of the discharge at any given location by means of dividing the discharge between a number of discharge points (e.g. spigots) has been shown to produce less scouring and steeper beach slopes.

Arguably the most efficient technique that might be adopted in order to increase the beach slopes at discharge is to increase the shear stress of the discharged tailings. This may be achieved for example by increasing the efficiency of the thickener to produce a higher underflow density; modifying the transport system to reduce shear stress losses, or potentially by modifying the structure of the tailings at discharge. 
A technique involving the in-line injection of a polymer into tailings at discharge is being investigated as a means of enhancing the release of supernatant water from tailings at discharge. An increase in yield stress at discharge due to expedited consolidation of the tailings may also facilitate an increase in beach slope. Further investigation will be required in order to ensure that this concept will be suitable for any given operation but proponents need to be aware of the costs for the polymer and upgraded management that will necessarily be associated with the technique.

\section{References}

Adkins, S.J. (2008) Underflow rheology optimisation to enhance thickener performance, in Proceedings 11th International Seminar on Paste and Thickened Tailings (Paste08), A.B. Fourie, R.J. Jewell, P. Slatter and A. Paterson (eds), 5-9 May 2008, Kasane, Botswana, Australian Centre for Geomechanics, Perth, pp. 83-95.

Bembrick, D. (2008) Impact of flocculant addition on tailings impoundment, ACARP Project C14064, Published October 2008.

Berger, A., Adkins, S., Hess, S., Flannagan, P. and Stocks, P. (2011) Step change improvements in underflow rheology, in Proceedings 14th International Seminar on Paste and Thickened Tailings (Paste2011), R.J. Jewell and A.B. Fourie (eds), 5-7 April 2011, Perth, Australia, Australian Centre for Geomechanics, Perth, pp. 135-153.

Daubermann, R.R. and Foldvari, Z. (2009) Evaluation of Ciba Rheomax chemical technology on ferrochrome deposits in concretelined tailings dams, in Proceedings 12th International Seminar on Paste and Thickened Tailings (Paste09), R.J. Jewell, A.B. Fourie, S. Barrera, J. Wiertz (eds), 21-24 April 2009, Viña Del Mar, Chile, Gecamin Limited, Santiago, Australian Centre for Geomechanics, Perth, pp. 39-46.

Jewell, R.J. (2010) Ensuring the credibility of thickening technology, in Proceedings 13th International Seminar on Paste and Thickened Tailings (Paste2010), R.J. Jewell and A.B. Fourie (eds), 3-6 May 2010, Toronto, Canada, Australian Centre for Geomechanics, Perth, pp. 23-32.

Kaiser, L.K., Lamperd, J.W., Loan, C. and Cooling, D.J. (2006) A new approach to bauxite residue dry stacking utilising Ciba Rheomax ETD technology, in Proceedings Ninth International Seminar on Paste and Thickened Tailings (Paste06), R.J. Jewell, S. Lawson and P. Newman (eds), 3-7 April 2006, Limerick, Ireland, Australian Centre for Geomechanics, Perth, pp. 57-67.

Li, A. (2011) Prediction of Tailings Beach Slopes and Tailings Flow Profiles, in Proceedings 14th International Seminar on Paste and Thickened Tailings (Paste2011), R.J. Jewell and A.B. Fourie (eds), 5-7 April 2011, Perth, Australia, Australian Centre for Geomechanics, Perth, pp. 307-322.

Matthews, J.G., Dhadli, N., House, P. and Simms, P. (2011) Field Trials of Thin Lift Deposition of Amended Mature Fine Tailings at the Muskeg River Mine in Northern Alberta, in Proceedings 14th International Seminar on Paste and Thickened Tailings (Paste2011), R.J. Jewell and A.B. Fourie (eds), 5-7 April 2011, Perth, Australia, Australian Centre for Geomechanics, Perth, pp. 271-280.

Robinsky, E.I. (1999) Thickened Tailings Disposal in the Mining Industry, E.I. Robinsky Associates Limited, Canada.

Seddon, K.D. and Fitton, T.G. (2011) Realistic Beach Slope Prediction and Design, in Proceedings 14th International Seminar on Paste and Thickened Tailings (Paste2011), R.J. Jewell and A.B. Fourie (eds), 5-7 April 2011, Perth, Australia, Australian Centre for Geomechanics, Perth, pp. 281-293.

Simms, P., Williams, M.P.A., Fitton, T.G. and McPhail, G. (2011) Beaching Angles and Evolution of Stack Geometry for Thickened Tailings - a Review, in Proceedings 14th International Seminar on Paste and Thickened Tailings (Paste2011), R.J. Jewell and A.B. Fourie (eds), 5-7 April 2011, Perth, Australia, Australian Centre for Geomechanics, Perth, pp. 323-338.

Wells, P.S., Revington, A. and Omotoso, O. (2011) Mature fine tailings drying-technology update, in Proceedings 14th International Seminar on Paste and Thickened Tailings (Paste2011), R.J. Jewell and A.B. Fourie (eds), 5-7 April 2011, Perth, Australia, Australian Centre for Geomechanics, Perth, pp. 155-166.

Williams, M.P.A., Murphy, S., MacNamara, L. and Khoshniaz, N. (2006) The Miduk Copper Project: Down-Valley Discharge of Paste Thickened Tailings, Design and Early Operating Experience, in Proceedings Ninth International Seminar on Paste and Thickened Tailings (Paste06), R.J. Jewell, S. Lawson and P. Newman (eds), 3-7 April 2006, Limerick, Ireland, Australian Centre for Geomechanics, Perth, pp. 117-130.

Williams, M.P.A., Seddon, K.D. and Fitton, T.G. (2008) Surface Disposal of Paste and Thickened Tailings - A Brief History and Current Confronting Issues, in Proceedings 11th International Seminar on Paste and Thickened Tailings (Paste08), A.B. Fourie, R.J. Jewell, P. Slatter and A. Paterson (eds), 5-9 May 2008, Kasane, Botswana, Australian Centre for Geomechanics, Perth, pp. 143-164. 